\title{
Predictive Validity of a Caries Risk Assessment Model at a Dental School
}

\author{
Emily Brons-Piche, George J. Eckert, Margherita Fontana
}

Abstract: Caries risk assessment (CRA) is an essential component informing clinical decision making for personalized caries management. The aim of this study was to assess the predictive validity of the CRA model used at the University of Michigan School of Dentistry. Data from patients treated in the school's adult integrated clinics between 2011 and 2014 who had a CRA documented were accessed from the electronic health record. Data extracted included caries risk category, date of reassessments, presence of caries risk indicators/factors, and treatments completed. Out of 2,449 patients who had a CRA completed in 2011, 447 had one or more reassessments at least 180 days after the initial CRA. Caries risk status was significantly associated $(p<0.0001)$ with the number of new caries-related treatments during follow-up, with significant increases $(p<0.01)$ in these needs for each caries risk level. For the 11,152 patients with a completed CRA in 2011-14, the prediction model (area under the curve $=0.82)$ included the following risk factors significantly $(\mathrm{p}<0.001)$ associated with increased caries experience/needs over time: stagnant plaque (OR 2.6, 95\% CI 2.4, 2.9), presence of salivary risk factors (OR 2.6, 95\% CI 2.2, 2.9), presence of dietary risk factors (OR 3.2, 95\% CI 2.9, 3.6), lack of adequate protective factors (OR 2.1, 95\% CI 1.8, 2.4), presence of conditions that affect compliance (OR 2.4, 95\% CI 1.9, 3.1), and past/current caries experience (OR 23.7, 95\% CI 21.1, 26.7). High risk patients were significantly more likely $(\mathrm{p}<0.0001)$ to have restorative, extraction, or endodontic treatments completed due to caries and less likely $(\mathrm{p}<0.0001)$ to have diagnostic procedures than low or moderate risk patients. These results suggest that the CRA model used at the dental school can help predict adult patients' future caries experience/needs.

Emily Brons-Piche, DDS, is a recent graduate of the School of Dentistry, University of Michigan; George J. Eckert, MAS, is a Biostatistician, Department of Biostatistics, School of Medicine, Indiana University; and Margherita Fontana, DDS, PhD, is Professor, Department of Cariology, Restorative Sciences, and Endodontics, School of Dentistry, University of Michigan. Direct correspondence to Dr. Margherita Fontana, Department of Cariology, Restorative Sciences, and Endodontics, School of Dentistry, University of Michigan, 1011 N. University, \#2393, Ann Arbor, MI 48109-1078; 734-647-1225; mfontan@umich.edu.

Keywords: dental education, dental caries, caries risk assessment, clinical skills, epidemiology

Submitted for publication 5/7/18; accepted 8/21/18

doi: 10.21815/JDE.019.017

$\mathrm{C}$ aries risk assessment (CRA) is an essential component of personalized oral health care delivery. CRA is defined as the process of establishing the probability of an individual to develop new carious lesions over a certain time period and/or the probability that there will be a change in severity and/or activity of currently present lesions. ${ }^{1}$ A CRA involves much more than just caries prediction; it also includes identifying factors that cause or increase/ decrease risk of disease to target cost-effective interventions to manage the caries disease process and remineralize carious lesions, as well as determining the periodicity of these services. ${ }^{2,3}$ Examples of interventions to be considered based on an individual's risk include fluoride toothpastes (over-the-counter and prescription), fluoride rinses, professionally applied fluoride products, sealants, and dietary and oral hygiene behavioral changes. ${ }^{2}$ Validation of a caries prediction tool involves longitudinal followup of caries-related changes over time (accounting for exposure to concurrent interventions to control disease), with the outcome expressed as continuous values (e.g., sensitivity, specificity, area under receiver operating characteristic curves [AUC]).
Because of the multifactorial and chronic nature of the dental caries disease process, studies on risk assessment tend to be complex, with multiple influences at the individual, family, and community levels challenging the prediction throughout the patient's lifetime. However, caries experience is still considered one of the greatest indicators of future risk. ${ }^{4,5}$ These variables are then generally taken together to develop a caries risk profile/category (low, moderate, or high risk), which needs to be reassessed over time. Numerous strategies and tools are available for CRA in daily practice, including an informal assessment, use of structured paper forms, and use of computerbased programs; but the majority are expert-based tools with limited validation. ${ }^{6}$ Today, the majority of structured paper CRA forms available for U.S. use have not been validated in the U.S., except for the adult caries management by risk assessment (CAMBRA) form in limited population groups. ${ }^{7-9}$ Yet, risk assessment is an essential component of cariology education in dental curricula, ${ }^{10}$ and thus dental schools must include CRA models in their clinics that have not been validated in their own populations. 
Furthermore, although it has been recognized that a practitioner's subjective impression may have good predictive power for determining a patient's risk, this is not always factored into existing CRA forms. ${ }^{11}$ The CRA form implemented in 2011 at the University of Michigan School of Dentistry helps guide students' critical thinking decision making process to assign a caries risk status, which is not automatically generated because of limited evidence supporting algorithms for CRA in adults.

Most CRA forms, including our school's, use a similar list of factors to inform the assessment, including caries risk indicators (e.g., past and current experience of dental caries), risk factors (e.g., frequency of fermentable carbohydrates, hyposalivation), and protective factors (e.g., exposure to effective interventions for caries management). These factors are associated with caries prevalence and/ or incidence, ${ }^{12}$ but their validity when used in multivariable forms with adult patients requires further evaluation in diverse adult populations. In addition, the school's CRA tool has skip-logic, and very few questions are initially displayed, with subsequent questions appearing only based on positive responses. The interactive form is completed by the student, and the last task is to assign the risk, which leads students to use not only information collected in the form, but also their clinical impression of the patient, factoring in their educational experiences/use of evidence. The school's definitions of risk are primarily based on presence and history of carious lesions, risk factors, and a critical analysis of the likelihood of disease progressing rapidly in the next couple of years. In general, a low caries risk is assigned to patients with no active carious lesions and no risk factors or a long history of risk factors that have not resulted in any lesions; a high caries risk is assigned to patients with active lesions (either multiple or progressing rapidly); and moderate risk patients are those with risk factors present but few carious lesions or very slowly progressing disease. ${ }^{13}$

The educational experiences at our dental school associated with CRA include year-long cariology training in the D1 year, as part of two semester courses, covering competencies and aspects of cariology as described in the U.S. cariology curriculum framework..$^{10}$ These include didactic, laboratory, and clinical experiences (both individually and in groups) on detection, diagnosis, risk assessment, prevention, and management of dental caries, to foster critical thinking and use of best evidence to solve clinical problems and cases throughout the life spectrum (children to elderly) associated with dental caries. In the clinics, D2-D4 students are required to complete the CRA and to develop and implement a caries management plan for every new patient, reassess it over time, and successfully pass every year a test case evaluation in clinic. Finally, every graduating student must pass the cariology section of an objective structured clinical examination (OSCE) in the D4 year that covers caries detection, risk, and management. In addition, there are yearly training and/or calibration sessions for faculty members associated with different aspects of cariology instruction.

The aim of this study was to assess the predictive validity of the CRA model used at the University of Michigan School of Dentistry. This aim was accomplished by addressing the following three objectives: 1) to assess if the CRA model was effective in predicting future overall caries experience/needs based on the initial caries risk status assigned;2) to assess the relationship between the initially assigned caries risk status and the types and number of treatments needed over time; and 3) to assess which risk factors were associated with an increased caries risk in the school's population.

\section{Methods}

The University of Michigan Institutional Review Board approved the study (HUM00103215). Data were accessed electronically from the University of Michigan School of Dentistry's patient health care record, axiUm (Exan, Las Vegas, NV, USA), using the software's data extraction based on defined criteria for patients treated between January 1, 2011 and December 31, 2014. Only patients who were treated in the vertically integrated adult clinics (VICs) were included $(n=25,416)$. We found that $42.5 \%$ of patients seen at the school between 2011 and 2014 had a completed CRA (11,152 patients).

To assess if the school's CRA tool was effective in predicting future caries experience, the patient pool for data extraction consisted of those who had a documented CRA in $2011(\mathrm{n}=2,449)$, with at least one follow-up appointment between 2012 and 2014 $(n=812)$. A total of 362 patients had to be excluded for the following reasons: 189 did not have a follow-up exam at least 180 days after the initial CRA (inclusion requirement); 103 charts were locked due to bad debt, 14 due to broken appointments, and seven due to non-compliance; 46 patients became edentulous during the study; and six charts were inaccessible 
for unknown reasons. Therefore, 447 patients with a completed CRA in 2011 and at least one follow-up CRA reassessment 180 days later (as most treatments are anecdotally completed in the school's clinics within this time frame after an initial appointment) were assessed to determine the number of new carious lesions developed to the end of 2014.

The patient's records were manually accessed by a single author (EBP) in the axiUm system, and the data extracted were de-identified. Data included gender, age, date of initial CRA and risk status, date of follow-up assessments, and number of new teeth with carious lesions. Although we refer in this article to "new carious lesions," dental procedures such as restorations and extractions with an associated diagnosis of caries were used as a surrogate for carious lesion experience, due to concerns with accuracy and completeness of charted carious lesions over time during this initial period.

The total follow-up time and total number of new lesions over time after the initial CRA was completed were calculated. A tooth with a new carious lesion was counted when any new procedure/ treatment with an associated diagnosis of caries was completed at least 180 days after the initial CRA. These procedures included restorations (e.g., using composite, amalgam, inlay, onlay, or full cuspal coverage) unless a specific diagnosis of non-caries (e.g., attrition, erosion, fracture) was recorded. In addition, they included extractions, root canals, or pulpectomies completed with a diagnosis of caries assigned. Treatments such as bleaching and veneers were not included as they were unlikely to be associated with caries.

A negative binomial regression model for count data was used to compare the total number of new lesions over time among the three caries risk levels (low, moderate, and high), while accounting for varying follow-up times. Gender and age were included in the model as covariates. A 5\% significance level was used for all tests.

To assess the relationship between number and types of treatment needs associated with caries over time and a patient's initial risk, only patients who had a CRA completed and treatments documented with a diagnosis of caries between 2011 and 2014 were evaluated $(n=3,115)$. The treatment codes were used to define each treatment as diagnostic, endodontic, extraction, fixed prosthodontics, periodontics, preventive, removable prosthodontics, restorative, or miscellaneous. Caries risk levels were compared for differences in the presence of procedures in each category using logistic regression and for differences in the number of procedures in each category using negative binomial regression for count data. Examples of diagnostic procedures were treatments such as observe/monitor tooth, radiographs, and oral exam. Preventive procedures included adult prophylaxis, fluoride application, oral hygiene instruction, and sealants. The miscellaneous category included consultation, nitrous oxide use, occlusal bitesplint, and post-treatment exam.

To analyze the risk factors associated with an increased caries risk, we assessed all patients with a documented CRA in the selected time frame $(n=11,152)$. In axiUm, the student must make a subjective decision on assigning the risk before developing a patient-centered risk management plan. To enhance use, the caries risk form initially has only two questions that appear in the chart: "Does the patient have any signs of caries experience?" and "Are factors present related to recent caries experience or increased risk?" Positive responses will cause more questions to appear. When a student indicates that there are factors present related to recent caries experience or increased risk, an additional screen appears with the following factors that can be selected: stagnant plaque in caries-susceptible sites, saliva, diet, inadequate protective modifying factors, and conditions that affect compliance. A positive response to each of these risk factors will prompt more questions and details. There is a final tab after risk is assigned to which the student assigns a recall interval and develops a caries management plan; however, management information was not evaluated in this study.

Each risk factor was compared to the caries risk level individually using Mantel-Haenszel chi-square tests. The number of risk factors was compared to caries risk level using cumulative logistic regression. A classification tree using recursive partitioning and multivariable logistic regression were used to evaluate combinations of risk factors to predict caries risk level.

\section{Results}

After initial CRA, the average follow-up times ranged from 2.1 years for high risk patients to 2.3 years for moderate and low risk patients, with $54 \%$ of patients classified as high caries risk (Table 1). Caries risk level was significantly associated $(\mathrm{p}<0.0001)$ with the number of new lesions during follow-up, with significant increases $(p<0.01)$ in the number of 
new lesions between each caries risk level (low vs. moderate, low vs. high, moderate vs. high). Neither age $(p=0.10)$ nor gender $(p=0.73)$ was significantly associated with number of new lesions during follow-up.

Risk level was significantly associated with the presence of new lesions during follow-up $(\mathrm{p}<0.0001$; Table 2). Number of new lesions was categorized as yes/no using three thresholds: $\geq 1$ or $\geq 2$ or $\geq 3$. The low and moderate risk groups were significantly different from the high risk group $(p<0.001)$ regardless of which threshold was used, but the low and moderate risk groups were not significantly different from each other ( $p=0.51$ for low vs. moderate $\geq 1$ new lesion, $\mathrm{p}=0.55$ for low vs. moderate $\geq 2$ new lesions, $\mathrm{p}=0.16$ for low vs. moderate $\geq 3$ new lesions). Thus, patients assigned to the high risk category were more likely to have greater than 1,2, or 3 new lesions at the end of the follow-up period than moderate and low risk patients. Interestingly, $41 \%$ of patients classified as low risk had at least one new lesion at follow-up, and $10 \%$ had more than three new lesions.

High risk patients had the highest number of treatment needs due to caries for each procedure category (Table 3 ). The highest percentages of those treatments were diagnostic, followed closely by restorations and then extractions. This pattern was the same for the moderate and low risk groups; however, the percentage of diagnostic procedures was higher in these last two groups $(91 \%$ of low risk patients and $81 \%$ of moderate risk patients had a diagnostic procedure completed).

High risk patients had significantly $(\mathrm{p}<0.05)$ more restorative, extraction, endodontic, preventive, or removable prosthodontic procedures than low risk patients (Table 3). In addition, high risk patients had significantly $(\mathrm{p}<0.05)$ more restorative, extraction,

\section{Table 1. Number of patients in each risk category and their characteristics}

\begin{tabular}{|c|c|c|c|c|}
\hline Variable & All & Low & Moderate & High \\
\hline Patients & 447 & $93(21 \%)$ & $112(25 \%)$ & $242(54 \%)$ \\
\hline Female, N (\%) & $236(53 \%)$ & $58(62 \%)$ & $65(58 \%)$ & $113(47 \%)$ \\
\hline Age: Mean (SD) & $56.6(18.5)$ & $60.4(17.0)$ & $60.5(18.9)$ & $53.4(18.3)$ \\
\hline Follow-up time in years: Mean (SD) & $2.2(0.8)$ & $2.3(0.8)$ & $2.3(0.8)$ & $2.1(0.8)$ \\
\hline Number of new lesions: Mean (SD) & $2.6(3.4)$ & $1.4(1.7)$ & $1.9(2.3)$ & $3.3(4.1)$ \\
\hline
\end{tabular}

Table 2. Percentage of patients in each caries risk category based on number of caries lesions developed during follow-up period

\begin{tabular}{lccc} 
Number of New Lesions & High Caries Risk & Moderate Caries Risk & Low Caries Risk \\
\hline$\geq 1$ new lesion & $65 \%$ & $46 \%$ & $41 \%$ \\
$\geq 2$ new lesions & $45 \%$ & $23 \%$ & $20 \%$ \\
$\geq 3$ new lesions & $32 \%$ & $15 \%$ & $10 \%$
\end{tabular}

Note: Relative rates were adjusted for different follow-up times. Number of new lesions were categorized as yes/no: $\geq 1$ or $\geq 2$ or $\geq 3$.

Table 3. Percentage of patients in each caries risk category who had a completed procedure type due to caries diagnosis

\begin{tabular}{|c|c|c|c|}
\hline Procedure Type & Low Caries Risk & Moderate Caries Risk & High Caries Risk \\
\hline Diagnostic & $399(91 \%)^{a}$ & $671(81 \%)^{b}$ & $1127(61 \%)^{\mathrm{c}}$ \\
\hline Restorative & $50(11 \%)^{\mathrm{a}}$ & $251(30 \%)^{b}$ & $1014(55 \%)^{c}$ \\
\hline Fixed prosthodontics & $1(<1 \%)$ & $3(<1 \%)$ & $11(1 \%)$ \\
\hline Extraction & $8(2 \%)^{a}$ & $33(4 \%)^{b}$ & $365(20 \%)^{c}$ \\
\hline Endodontics & $0^{a}$ & $1(<1 \%)^{a}$ & $22(1 \%)^{b}$ \\
\hline Preventive & $9(2 \%)^{a}$ & $36(4 \%)^{b}$ & $99(5 \%)^{b}$ \\
\hline Removable prosthodontics & $1(<1 \%)^{a}$ & $0^{\mathrm{ab}}$ & $35(2 \%)^{b}$ \\
\hline Periodontics & $1(<1 \%)^{\mathrm{ab}}$ & $2(<1 \%)^{\mathrm{a}}$ & $22(1 \%)^{b}$ \\
\hline Miscellaneous & $3(1 \%)$ & $6(1 \%)$ & $5(<1 \%)$ \\
\hline
\end{tabular}

Note: Groups with different superscript letters were significantly different at $\mathrm{p}<0.05$. 
endodontic, or periodontic procedures than moderate risk patients, but significantly fewer diagnostic procedures. Moderate risk patients had significantly $(p<0.05)$ more restorative, extraction, or preventive procedures completed due to caries than low risk patients. Low risk patients had significantly $(\mathrm{p}<0.05)$ more diagnostic procedures than both moderate and high risk patients.

Patients with low and moderate risk were not significantly different for the presence of at least one fixed prosthodontic $(\mathrm{p}=0.69)$, endodontic $(\mathrm{p}=1.00)$, removable prosthodontic $(\mathrm{p}=1.00)$, periodontics $(\mathrm{p}=0.96)$, or miscellaneous $(\mathrm{p}=0.94)$ procedure. $\mathrm{Pa}-$ tients with low and high risk were not significantly different for the presence of at least one fixed prosthodontic $(p=0.35)$, periodontic $(p=0.10)$, or miscellaneous $(\mathrm{p}=0.21)$ procedure. Patients with moderate and high risk were not significantly different for the presence of at least one fixed prosthodontic $(\mathrm{p}=0.44)$, preventive $(\mathrm{p}=0.25)$, or miscellaneous $(\mathrm{p}=0.11)$ procedure.

Regarding risk factors associated with an increased caries risk, stagnant plaque in cariessusceptible sites, hyposalivation, dietary risk factors, inadequate protective modifying factors, conditions that affect compliance, and signs of caries experience were significantly associated with increased caries risk (Table 4). The multivariable logistic regression model for caries prediction had an $\mathrm{AUC}=0.82$ and included all six significant factors $(\mathrm{p}<0.0001)$ : stagnant plaque (Odds Ratio [OR] 2.6, 95\% Confidence Interval [CI] 2.4, 2.9), salivary risk factors (OR 2.6, $95 \%$ CI $2.2,2.9)$, dietary risk factors $(\mathrm{OR}=3.2,95 \%$ CI 2.9, 3.6), lack of protective factors (OR 2.1, 95\% CI 1.8, 2.4), conditions that affect compliance (OR $2.4,95 \%$ CI 1.9, 3.1), and caries experience (OR 23.7, 95\% CI 21.1, 26.7). A higher number of risk factors was significantly associated with increased risk categories ( $\mathrm{p}<0.0001$, OR 4.6, 95\% CI 4.4, 4.8 for each additional risk factor) (Table 5).

\section{Discussion}

This study assessed the validity of the CRA model soon after implementation in our clinics. These data will help the school assess changes over time to our risk-based caries management and educational model. The CRA model was built with skip-logic to enhance use, based on focus groups in which faculty and students reported extended length/time to complete forms was a deterrent. Soon after launch, almost $43 \%$ of charts had CRA forms completed. Quality assessment data showed that, in the last two years, $80-88 \%$ of new patients have completed CRA. At the time of implementation of this form, there were no other validated forms for use with adults in the U.S. Since then, the CAMBRA form has been studied for

Table 4. Presence of risk factors for each caries risk group

\begin{tabular}{lccc} 
Risk Factor & $\begin{array}{c}\text { Low } \\
(\mathrm{n}=2139)\end{array}$ & $\begin{array}{c}\text { Moderate } \\
(\mathrm{n}=2917)\end{array}$ & $\begin{array}{c}\text { High } \\
(\mathrm{n}=6096)\end{array}$ \\
\hline (Stagnant) plaque in caries-susceptible sites & $122(6 \%)$ & $745(26 \%)$ & $2491(41 \%)$ \\
Saliva-related risk factors & $64(3 \%)$ & $323(11 \%)$ & $1111(18 \%)$ \\
Diet-related risk factors & $80(4 \%)$ & $511(18 \%)$ & $2375(39 \%)$ \\
Inadequate protective modifying factors & $66(3 \%)$ & $353(12 \%)$ & $1387(23 \%)$ \\
Conditions that affect compliance & $18(1 \%)$ & $100(3 \%)$ & $444(7 \%)$ \\
Signs of caries experience & $543(25 \%)$ & $2381(82 \%)$ & $5967(98 \%)$
\end{tabular}

Note: All risk factors were significantly $(\mathrm{p}<0.0001)$ associated with increased caries risk.

Table 5. Mean number of caries risk factors based on caries risk status

\begin{tabular}{|c|c|c|c|c|c|c|}
\hline \multirow[b]{2}{*}{ Caries Risk Category } & \multicolumn{6}{|c|}{ Number of Risk Factors } \\
\hline & $\mathrm{N}$ & Mean & SD & SE & Min & Max \\
\hline Low & 2139 & 0.417 & 0.668 & 0.014 & 0 & 4 \\
\hline Moderate & 2917 & 1.513 & 0.903 & 0.017 & 0 & 6 \\
\hline High & 6096 & 2.260 & 1.024 & 0.013 & 0 & 6 \\
\hline
\end{tabular}


its reliability in identifying patients at increased risk in predoctoral adult clinics at the University of California, San Francisco (UCSF) ${ }^{7-9}$ The accuracy of the CAMBRA form in children compared to other CRA forms in a population in Hong Kong found limited accuracy. ${ }^{14}$ One study using the CAMBRA form with adults suggested that "low-risk and moderate-risk categories may not be sufficiently and distinctively different in predicting increasing risk of future caries" (p. 198).${ }^{15}$ In our study, we found similarly that we could not separate the prediction of caries in moderate and low risk patients, even when using different thresholds for caries experience needs over time.

One limitation of this study was the lack of consistent detailed diagnostic information for carious lesions during the initial CRA. Although students learn use of the International Caries Detection and Assessment System (ICDAS) for assessment of carious lesion severity and activity, and collapsed ICDAS codes are used in clinic (non-cavitated and cavitated lesions, active and arrested), our study found that these diagnostic codes were not being consistently used. Although in many cases the lesion diagnosis may have been written into a treatment note, written notes were not included for the data extraction in this study. Without detailed lesion severity and activity charting at initial and follow-up exams, treatment completed associated with a caries diagnosis was used as a surrogate for caries experience. This practice calls attention to the importance of reinforcing lesion charting over time in educational and clinical activities.

In another limitation, it is possible that there were treatments that were not coded appropriately and thus missed, and it is also possible that treatments completed due to trauma, periodontal condition, or replacement of previously missing teeth were miscoded. A similar limitation was noted in a study conducted at UCSF, where investigators analyzed decayed and filled teeth but noted that the increase in filled teeth may have been due to reasons other than carious lesions. ${ }^{8}$

Regarding defining the time frame for the follow-up exams, these were considered as the next documented Periodic, Update Plan, or Limited Oral Exams visit, as long as it was completed at least 180 days following the initial CRA. This criterion was put in place based on the assumption (based on historical clinical data observed during data extraction) that most treatment would be completed within 180 days of a treatment plan. Another limitation of this study is that the data extraction method did not allow for determination of when the treatment was originally planned-only when it was completed. Another limitation is that our analyses did not account for dental treatment due to caries that could have been completed outside of the dental school. Although unlikely, as these were patients of record in the school with follow-up visits, this possibility cannot be ignored.

While the data from this study suggest that the CRA model is helpful to assess future caries needs, it is important to mention that $41 \%$ of low-risk patients still had at least one new lesion at follow-up exams. This finding was similar in the study conducted at UCSF, where nearly half of the low-risk patients were found to have a carious lesion at follow-up exams. ${ }^{8}$ Those researchers suggested that the CRA was a useful tool for risk stratification, which is similar to our findings in which patients experienced a significantly higher percentage of new caries-related treatments based on increasing categories of risk. Due to the larger number of low and moderate risk patients returning with dental caries-associated treatment needs, it is evident that further studies regarding our caries management plans and their implementation are needed. After the caries risk is assigned, the following tab in our caries risk assessment form is the caries management plan, where students document the proposed plan agreed upon with the patient. The implementation of these plans is difficult to assess because details are often provided in treatment notes, and thus data extraction is time-consuming. This difficulty was also noted in CAMBRA's system when evaluated at UCSF. ${ }^{16}$

The risk indicators and factors from the CRA form that were significantly associated with future caries experience/needs were current/past caries experience, presence of stagnant plaque in cariesprone areas, hyposalivation, frequent consumption of fermentable carbohydrates, lack of protective modifying factors, and presence of conditions that affect compliance. In considering individual odds ratios, caries experience was the greatest factor associated with an increased caries risk, followed by stagnant plaque, which is similar to what was found in a previous study. ${ }^{17}$ Overall, we did not find that a single factor placed patients into a certain risk category; rather, the number of factors associated with risk increased as the level of risk was elevated. On the other hand, as risk was not predetermined but assigned, it is likely that patients with caries experience (especially current experience) who also had identified causative factors and lack of exposure to effective interventions were assigned a higher risk 
category by students. The dental school's CRA form does not generate a risk directly based on answers provided to the practitioner (other than a low risk is precluded if caries activity is present in the mouth); rather, the practitioner must critically think about all of the information gathered throughout the exam to assign the risk. An evaluation of the thought process behind assigning risk based on the factors present would be interesting and could lead to better understanding of a patient's placement into a specific risk category and assist in the assessment of students' critical thinking skills. ${ }^{17-19}$

\section{Conclusion}

This study found that the dental school's CRA tool when used by students was able to identify patients at increased risk of needing treatment associated with caries over time; however, the evidence suggests that, for prediction purposes, the tool is best at helping draw a distinction between regular risk (low/moderate) and increased risk (high risk). Caries treatment needs over time were significantly associated with the initial caries risk level assigned, with higher risk patients having significantly higher caries experience/needs than lower risk patients. In addition, higher risk patients had more restorative, extraction, and endodontic procedures completed over time than low or moderate risk patients. With an increased risk status, there was a greater number of associated caries risk factors.

\section{Disclosure}

The authors declared that they have no conflicts of interest related to this research.

\section{Acknowledgments}

We would like to thank the following staff from the University of Michigan School of Dentistry: Jean Thompson for many hours of assistance in running reports and extracting clinical data from axiUm, and Emily Yanca for support in obtaining IRB exemptions and approval.

\section{REFERENCES}

1. Fontana M. The clinical, environmental, and behavioral factors that foster early childhood caries. Pediatr Dent 2015;37:217-25.
2. Young DA, Featherstone JD. Implementing caries risk assessment and clinical interventions. Dent Clin 2010;54(3):495-505.

3. Jenson L, Budenz AW, Featherstone JD, et al. Clinical protocols for caries management by risk assessment. J Calif Dent Assoc 2007;35(10):714-23.

4. Twetman S, Fontana M, Featherstone JD. Risk assessment: can we achieve consensus? Community Dent Oral Epidemiol 2013;41(1).

5. Ditmyer MM, Dounis G, Howard KM, et al. Validation of a multifactorial risk factor model used for predicting future caries risk with Nevada adolescents. BMC Oral Health 2011;11(1):18.

6. Tellez M, Gomez J, Pretty I, et al. Evidence on existing caries risk assessment systems: are they predictive of future caries? Community Dent Oral Epidemiol 2013;41(1):67-78.

7. Featherstone JD, Chaffee BW. The evidence for caries management by risk assessment (CAMBRA). Adv Dent Res 2018;29(1):9-14.

8. Chaffee BW, Cheng J, Featherstone JD. Baseline caries risk assessment as a predictor of caries incidence. J Dent 2015;43(5):518-24.

9. Doméjean S, White JM, Featherstone JD. Validation of the CDA CAMBRA caries risk assessment: a six-year retrospective study. J Calif Dent Assoc 2011;39(10):709-15.

10. Fontana M, Guzmán-Armstrong S, Schenkel AB, et al. Development of a core curriculum framework in cariology for U.S. dental schools. J Dent Educ 2016;80(6):705-20.

11. Twetman S, Fontana M. Patient caries risk assessment. In: Detection, assessment, diagnosis and monitoring of caries, vol. 21. Basel, Switzerland: Karger Publishers, 2009:91-101.

12. Fontana M, Gonzalez-Cabezas C. Minimal intervention dentistry: part 2, caries risk assessment in adults. Br Dent J 2012;213(9):447-51.

13. Fontana M, Zero DT. Assessing patients' caries risk. J Am Dent Assoc 2006;137(9):1231-9.

14. Gao X, Wu ID, Lo EC, et al. Validity of caries risk assessment programs in preschool children. J Dent 2013;41(9):787-95.

15. Tellez M, Bhoopathi V, Lim S. Baseline caries risk assessment using CAMBRA may predict caries only in high and extreme caries risk groups. J Evid Based Dent Pract 2015;15(4):197-9.

16. Chaffee BW, Featherstone JD. Long-term adoption of caries management by risk assessment among dental students in a university clinic. J Dent Educ 2015;79(5):539-47.

17. Doméjean S, Léger S, Rechmann P, et al. How do dental students determine patients' caries risk level using the caries management by risk assessment (CAMBRA) system? J Dent Educ 2015;79(3):278-85.

18. Fontana M, Gonzalez-Cabezas C, de Peralta T, Johnsen D. Dental education required for the changing dental environment. J Dent Educ 2017;88(8 Suppl):eS153-61.

19. Guzman-Armstrong S, Warren JJ, Cunningham-Ford MA, et al. Concepts in critical thinking applied to caries risk assessment in dental education. J Dent Educ 2014;78(6):914-20. 